\title{
PATRONIMICI IN -IČ A TRIESTE NEL BASSO MEDIOEVO
}

Operando lo spoglio delle forme antroponimiche che compaiono in Trieste nei secoli $X I I I^{\circ}, X I V^{\circ}$ e $X V^{\circ}$ sia da fonti originali che da fonti già pubblicate e da alcune Tesi di laurea in Storia medioevale, siamo incorsi finora in $18 \circ 19$ patronimici slavi in -ič portati da una cinquantina di persone. Li abbiamo trovati nelle seguenti fonti:

SSMM Pergamene, registri e atti cartacei (atti di donazione, testamenti, urbari, contratti di affittanza ecc.) del convento benedettino dei Santi Martiri, limitatamente ai tre secoli studiati; Archivio di stato in Trieste, segn. C. R. S. Intendenza Commerciale, buste 664-677.

Registro cartaceo del presbiter Iohannes (l'apporto di altre mani è insignificante) con l'elenco delle entrate e delle uscite (in derrate e danaro) del Capitolo triestino per il periodo 1315-1320; Archivio capitolare, segn. 2 D 4.

$\mathrm{C}$

Registro cartaceo contenente il computo della quantita di cera da candele incassata dai canonici canipari del Capitolo a titolo di diritto di stola nera per il periodo 1356-1376; Archivio capitolare, segn. BAA 15.

M

Don Angelo Marsich, Regesto delle pergamene conservate nell'Archivio del Reverendissimo capitolo della Cattedrale di Trieste, in: Archeografo triestino, 1877-85, limitatamente al periodo preso in esame.

Luigi Parentin, Regesto di 85 documenti inediti dell' Archivio Capitolare di Trieste, in: Archeografo triestino 1965-66, pagg. 147-189, limitatamente al periodo preso in esame.

Poche altre fonti secondarie saranno riportate con il titolo per intero.

Per una comparazione diacronica del materiale antroponimico che tratteremo ci riferiremo a:

PIz Aldo Pizzagalli, per l'italianità dei cognomi nella Provincia di Trieste, Trieste 1929.

TEL SIP Società Italiana per l'Esercizio Telefonico p. a., Trieste e Provincia 1982-83, elenco ufficiale alfabetico... degli abbonati al telefono aggiornato al 31 marzo 1982 . 

Ljubljana 1974.

Per il fondo antroponimico slavo infine ci riferiremo a: PN Franz Miklosich, Die Bildung der slavischen Personennamen, Heidelberg 1927.

Ogni altro riferimento bibliografico sarà citato per intero.

Il primo patronimico in -ič compare in SSMM 1283 con l' ablativo latino Manesclavo filio Almerici Cucich e sembra già cognominizzato. Lo ritroveremo in M 1516 Colota, figlia ed erede del fu ser Giovanni cigoti e moglie di ser Michele de zucich e questo dato $\mathrm{ci}$ consente di stabilire l'esatta dizione della prima forma nominale: cučič, suffragata dall'esistenza del cognome in ZSSP Cučič a Gorizia; le forme cognominali TEL Cucich (2x), zuzich $(4 \cdot \mathrm{x})$ e zuzig $(2 \mathrm{x})$ non ci possono offrire dati sicuri di paragone per possibili e probabili interferenze con altre forme cognominali (ZSSP: Kučič, žužic̆); per la forma Cuciz attestata attualmente a Nimis (cfr. P. Merkù, slovenski prijmki na zahodni meji, Trst 1982) è altrettanto difficile stabilire $1^{\prime}$ esatta dizione originale.

Dall'inizio del' 300 i patronimici in -ič sono frequenti: in Q 1315-1320 è citato dieci volte un Martinus Babic che nel 1316 compare una volta nella forma a Martino Babic, 1316-1320 è citato quattro volte un $a b$ Almerico Babic. In seguito, fino alla fine del '400, il nome compare altre volte nelle forme (nomen) Babich (19 x), abl. Martino Babicho, (nomen) Babic (3 x), Aymericus Babis...Aymerici Babici, filie odorici Babigh, Simone de Babich. Il cognome compare frequentissimo pure in seguito e si collega con PIZ Babich>Balbi (pag. 128), Babic (2 x), Babich (4 x)>Babbi (l x), Balbi (l x), Babini (3 x), Babi (l x, pag. 181) e con TEL Babic (21 x), Babich (75 x), Babici (21 x), Babbini ( $1 \mathrm{x})$, Babini $(7 \mathrm{x}), \operatorname{Balbi}$ (108 $\mathrm{x}$ ). Evidente ne è fin dall'inizio la funzione cognominale con un'unica possibile eccezione per quel Dominicus che una volta sola è detto gener babic in virtù dell'esistenza di un più influente suocero dal cognome 
Babič. Di rilevante interesse è la forma $B a b i s$ che avvalla la nostra supposizione sull'interferenza dei formati -ic $e-i s$ lungo il confine linguistico slavo-romanzo, espressa in slovenski pxïimki nä zahodni meji, già citati, s. v. Menič e Menis.

Dai dati finora esposti sembra già sufficientemente chiara la funzione cognominale dei patronimici in -ič fin dal loro apparire in Trieste e tale funzione è suffragata dalla maggior parte dei dati che seguono. In Q 1317-19 compare $6 \times$ a Leo $(n)$ Minic senza altri riscontri coevi nè diacronici.

In Licia Persi-Ricci Cocevar, I registri dei notai triestini dei malefici facina de Canciano e Jacobus Gremon (1352 e 1354), Tesi di laurea in Storia medioevale, Università di Trieste, a.a. 1979-80, compare nel $13545 \mathrm{x}$ - sempre con l'identica forma scritta - il cognome vrauxmich, riferito due volte a un crismanus, tre a un simon: non ho potuto finora consultare l'originale nè posso valermi di riscontri sincronici e diacronici.

In C 1359 leggiamo per mortem Dominici Boxichi e lo colleghiamo con PIZ Bosich $(4 \mathrm{x})>$ Bossi. $(3 \mathrm{x})$, Natali (1 x, pag. 191) e Bozic $(4 \mathrm{x})>\operatorname{Bozzi}(1 \mathrm{x})$, Bossi $(3 \mathrm{x}$, pag. 19l) e con TEL Bosic $(1 \mathrm{x})$, Bosich $(23 \mathrm{x})$, de Bosichi ( $2 \mathrm{x})$, Bosick (1 x), Bosig ( $1 \mathrm{x})$, Bosi $(4 \mathrm{x})$, Bossi $(171 \mathrm{x})$, Bozzi $(7 \mathrm{x})$, Bosini $(3 \mathrm{x}) \mathrm{e}$ forse qualche altra forma recentemente italianizzata.

In $P 1359$ si menziona una casa di Jure stipich in contrata Castelli.

In M 1366 si nomina il fu Giorgio de sosich di Trieste, cfr. PIZ Sosic $(11 \mathrm{x})$, sosich $(2 \mathrm{x})$, $\operatorname{sossich}(7 \mathrm{x})>\operatorname{sossi}(19 \mathrm{x})$, Sossini (1 $x$, pagg. 313-14) e TEL $\operatorname{sosic}(16 x), \operatorname{sossich}(5 x)$, sossi $(169 \mathrm{x})$, sossini $(4 \mathrm{x})$.

In C 1370 leggiamo pro morte Iuri sipich senza riscontri sincronici e diacronici.

In C 1395 leggiamo per mortem... Ganci de Brentarich, pure senza ulteriori riscontri.

In Attilio Hortis, Di Santo dei pellegrini e di Blenghio dei Grilli, lettera a Carlo Combi, in: Archeografo triestino 1881-82, pagg. 399-443, leggiamo a nobili domina Benevenuta uxore relicta nobilis viri Antonij de Wesnuich senza altri riscontri. 
In C 1404 leggiamo pro morte Andree de Cholubich e in SSMM, alla stessa data, vinea Andree Golubich, tenet Andrea Golobich, e nel 1451 ser Andree Colobich: dalle quattro forme di scrittura differenti, riferite tutte alla stessa persona, si può desumere con sufficiente sicurezza la dizione originale Golobic con seriore passaggio dialettale $o>$ u e si deve constatare la presenza contemporanea di una forma corrotta Kolobič/Kolubič. Tutte e quattro le forme sono conservate in PIZ Colobig (pag. 132), Golobig (136), Colubig (3 x, pag. 203), Golubig (pag. 222), tutte italianizzate in Colombi; in TEL compare soltanto colubig (2 x).

In M 1420 stefanus susic va riferito a PIZ susig, sussich, Sussig > sussi (pag. 156) e sussich (3x) > Suzzi (2x), succi (1 $x$, pag. 320) e a TEL susic (1 x), susich (1 x), sussich $(2 \mathrm{x})$, susig $(1 \mathrm{x})$, sussig $(2 \mathrm{x})$, sussi $(8 \mathrm{x})$, succi $(10 \mathrm{x})$ e forse $s u-$ si $(6 \mathrm{x})$.

In SSMM 1421 in contrata Grondelera subtus montem... vinea Ianes scerlich va riferito all'attuale forma cognominale TEL scherlic $(2 \mathrm{x})$, scherlich $(13 \mathrm{x})$, skerlic (l x) e letto sk(e)rlíč o skk(e)rlič; il cognome non è documentato in PIZ e non è noto in ZSSP.

In M 1434 è menzionato un sacerdote Giorgio sbirlitsch senza riferimenti diacronici.

In M 1490 Antonio de Castelino detto Muchif, cittadino di Trieste. Nonostante quel "detto" pensiamo a una precisa funzione cognominale, suffragati in ció dalla tarda datazione e dalle attestazioni diacroniche: PIZ Muhic > Mosca (pag. 148), TEL Muhic (2 x), Muhich (1 x).

Rimangono tre casi isolati per $i$ quali dai dati a disposizione non si può evincere con certezza la funzione cognominale del patronimico, in un caso si può anche dubitare della sua appartenenza alla categoria dei patronimici in -ič.

M 1325 domus que fuit Venerande de obedif, senza altre attestazioni sincroniche e diacroniche, può essere letto obedic oppure obedic. SSMM 1344' (riferito al 1317?) penes vineam Cenchachig senza altri riscontri. C 1367 (per mortem) Volfic con attestazioni diacroniche in PIZ Volcic > Volsi ( $1 \mathrm{x}$, pag. 124), $\operatorname{volcich}(5 \mathrm{x})>\operatorname{Volsci}(1 \mathrm{x}), \operatorname{Volli}(4 \mathrm{x}, \operatorname{pag} .333) \mathrm{e}$ in 
TEL Volcic $(14 \mathrm{x}), \operatorname{Volcich}(1 \mathrm{x}), \operatorname{Volsi}(10 \mathrm{x}), \operatorname{Volli}(9 \mathrm{x})$. In questi tre casi possiamo trovarci di fronte a nomi personali, a patronimici o a cognomi, nel secondo caso forse anche a un soprannome. Ma l'esiguità di quest'ultima casistica ci concede tuttavia di poter affermare che $i$ patronimici slavi (preponderanti gli sloveni in -ić, sparuti $i$ croati in -ić) si diffondono in Trieste a partire dalla fine del'200, sono già numerosi all'inizio del' 300 e si attestano fin dal basso Medioevo con funzione cognominale; la sopravvivenza della funzione di nome personale (forse volčičr) è indimostrabile, come lo è la funzione patronimica.

II

La primaria funzione patronimica, espressa dal formante -ic (-ić), ci porta a cercare l'origine delle forme nominali (cognominali) elencate in primo luogo da nomi personali.

Derivano da nomi personali doppi di tradizione slava:

BOžič, tramite un ipocoristico Božo (o *Bogo), da nomi come Božidar, Božislav (o Bogodan, Bogolep, Bogomil)o simili; Cfr. PN 11 Bogŭ "deus" e C 1360 per mortem fratris Bosidari. Babič se da Baboneg. Altrimenti saremmo in presenza di un matronimico da PN 2 Baba "vetula, avia" con il significato di "chi è stato allevato (dopo la morte della madre) da una donna anziana (nonna, zia)".

Derivano da zooantroponimi slavi:

Golobič da PN 77 Golgbi "columba", cfr. SSMM 1421 petrus dictus Golop, vinea petri dicti Golop, evidentemente già cognominizzato, M 1360 donna Golobica figlia del fu Gerdine de Mocho, C 1362 (per mortem) Cholobice: nel personale femm. della metà del '300 osserviamo la fluttuazione tra $g$ e $k$ iniziali, già osservata nel patronimico preso in esame; cfr. ancora PIZ Golob $>$ Colombo (pag. 119), Golob, Gollob>Colombi (136), Gollob $(4 \mathrm{x})$, Golob $(\mathrm{I} \mathrm{x})>\operatorname{Colombi}(\mathrm{lx})$, Colombo $(3 \mathrm{x})$, Gollo (1 x, pag. 222) e TEL Golob $(7 \mathrm{x})$, Gollob (2 $\mathrm{x})$ e conseguenti forme italianizzate, che si confondono con forme cognominali italiane originarie omofone. 
Muhič da slov. Muha. "mosca", cfr. PIZ Muha> Mosca (pag. 148) e TEL Muha ( $8 \mathrm{x}$ ), Mucha ( $1 \mathrm{x}$ ), mentre la forma cognominale italianizzata si confonde con l'omofono cognome originario dal Trentino.

Volčič da PN 43 Vŭlkŭ "lupus",cfr. PIZ Vuk>Adamolli (pag. 118), Vuk>Vucini (124), Volk>Volli (2 x), Vouch>Vocchi $3 \mathrm{x})$, Vouk $(12 \mathrm{x})>\operatorname{Vucci}(4 \mathrm{x}), \operatorname{Lupi}(6 \mathrm{x})$, Vocchi (2x, pag. 333), $\operatorname{Vuk}(5 \mathrm{x})>\operatorname{lupi}(2 \mathrm{x})$, Lupo $(3 \mathrm{x}$, pag. 334) e TEL Volk (9 x), Vouch $(7 \mathrm{x}), \operatorname{Vouck}(1 \mathrm{x}), \operatorname{Vouk}(28 \mathrm{x}), \operatorname{Vovk}(7 \mathrm{x}), \operatorname{Vocch} i$ (3 x), volli $(9 \mathrm{x}), \operatorname{Vuch}(18 \mathrm{x}), \operatorname{vuk}(8 \mathrm{x})$, tralasciando altre forme italianizzate che si confondono con forme cognominali originarie italiane omofone.

Derivano, tramite forme ipocoristiche, da agionimi:

sipič, se da Joseph tramite (Jo)sip.

stipić da stephanus tramite il croato icavo stipe; ma con la forma nominale rilevata e la spiegazione da una forma nominale croata icava contrastano le attestazioni diacroniche in PIZ stepich>steffi (pag. 156), stepich> steppini (1 x, pag. 316) e in TEL stepich (1 x), stepini (1 x), steppini (2 x): queste ultime $c i$ permettono $d i$ supporre un ipocoristico sloveno *Stepa analogo al serbo-croato stijepa, cfr. PN 367 stefanŭ "Stephanus".

Minič se da Dominicus tramite un ipocoristico. *Min-.

Singolare ci sembia il patronimico da nome di mestiere Brentarič, ma non ci sorprende, poiché la quasi totalità dei brentar(i)us (fabbricatori o portatori di brente?) registrati in Trieste tra il 1308 e il 1455 portano nomi slavi (sloveni): Cergne, Vulfei, Mucha, gen. Hancegle, gen. Jachegli, Soubanus (anche se il personale deriva da *Salvanus, il cognome souban, suban vive esclusivamente in area slovena, è tipico della valle del Vipacco, del Carso triestino e della città), Nedelus dictus Glaviateq, Marince, Jachxe, Jachil, Pentachosta filius condam Janexi brentarii, Jose, uxoris permani, Iacxe, rarney, Crisman, oppure agionimi latini senza tratti caratteristici nazionali oppure, più raramente, nomi di origine tedesca (abl. Herimano, Herman, Anderlinus de Marpurgo) portati più o meno evidentemente da sloveni. 
Questa sommaria descrizione ci dà ragione di appena dieci patronimici in -ić (-ić) su diciotto o diciannove. Per i rimanenti possiamo azzardare qualche proposta di soluzione, ma desideriamo soprattutto segnalarli ad altri studiosi che possano contribuire alla loro spiegazione. Procedendo in ordine alfabetico:

per cenchachig non possiamo nemmeno proporre l'esatta dizione; che potrebbe suonare con l'iniziale $c-, c-, k-$, mentre per il aigramma ch possiamo oscillare soltanto tra le pronunce $\check{c}$ e $k$, ferma restando per la finale la pronuncia -c $(-c)$ : meno probabile, anche se non da escludersi del tutto, appare la pronuncia $-k$. Con tante variabili le possibilità di lettura sono numerose senza tener conto di potenziali errori di scrittura o di lettura e senza considerare la difficile, per ora impossibile classificazione del tipo nominale, privi come siamo di ogni riferimento sincronico e diacronico.

Per cučic bisognerà cercare una soluzione in una prospettiva molto più ampia che tenga conto di molte forme nominali affi$\mathrm{ni}$ in uno spazio molto più largo.

Per obedič (?) ci si può forse riferire al cognome ZSSP obed tipico dell'area slovena orientale o allo zooantroponimo zssP obad "tafano".

Per Sosič e susič sembra opportuno lasciare il campo sgombro a spiegazioni che tengano conto pure di una possibile derivazione istriana per almeno una delle due forme cognominali, investendo nella ricerca pure qualche specialista di antroponimia istriana.

Per Skrlič o škrlič la forma nominale va studiata anche in rapporto alla forma cognominale sk $(e) r i(j)$ ed altre più complesse, delle quali non abbiamo finora trovato traccia in Trieste nel basso Medioevo.

Per Vrauxmich, wesnuich e zbirlič (?) infine ogni congettura sembra inutile prima di aver controllato le fonti originali.

La certezza che $i$ patronimici in -ič $(-i c ́)$ si attestano in Trieste a partire dalla fine del '200 con funzione cognominale ci consente di porci in chiusura una domanda: sono questi $i$ 
primi cognomi in $-i \check{c}(-i c ́)$ attestati in area slovena (croata)? In base ai dati raccolti nel basso Medioevo triestino si puō datare - data la percentuale relativamente esigua di tali forme cognominali rispetto all'universo cognominale sloveno (croato) documentato nei tre secoli presi in esame-I'insorgenza delle prime forme cognominali slovene (croate) da patronimici in -ič (-ić) verso la fine del '200?

Le interrogazioni con le quali concludiamo la presente comunicazione desiderano stimolare la collaborazione interregionale senza la presunzione di aver stabilito nulla di definitivo.

\author{
Povzetek \\ IZ Oð̌TNEGA IMENA: IZPELJANI PRIIMKI NA -ǐ் V TRSTU \\ POZNEGA SREDNJEGA VEKA
}

Pri ekscerpiranju antroponimičnih oblik iz tržaških arhivskih virov v 13., 14. in 15. stoletju ter, za isto obdobje, tudi iz že objavljenih virov ter iz doktorskih disertacijo srednjeveški zgodovini pri tržaški univerzi smo naleteli na 18 ali 19 slovenskih patronimikov na -ič, ki jih je nosilo kakih 5o oseb. Analiza vseh pisnih oblik omogoča diahronično primerjavo z oblikami v 20. stoletju, kakor jih izpričjeta pizzagallijev seznam poitalijančenih priimkovnih oblik v tržaški provinci za fašizma in telefonski imenik za tržaško provinco 1982-83. Le manjše število patronimičnih oblik iz poznega srednjega veka je. usahnilo in ga ni najti $v$ sodobnih virih.

večino opisanih patronimikov na -ič (redkeje: -ić) je izpeljati iz predkrščnskih slovanskih osebnih imen, bodisi iz dvojnih imen oz. iz njihovih hipokoristikov, bodisi iz zooantroponimov, redkejše iz hagionimov oz. njihovih hipokoristikov, enega samega pa iz poklicnega imena. Točni ugotovitvi izvirnega imena, iz katerega je bil izpeljan patronimik, se izmuzne se peščica imen, bodisi da so zgodovinske priče preskromne, bodisi da imamo opraviti s pisnimi pogreski ali napakami pri branju. Vsekakor so slovenski patronimiki na -ič izpričani v Trstu od konca 13. stoletja in nam postavljajo vprasanje, ali so priimkovne oblike na -ič nastale v Trstu v tem času in se odtod razsirile na vesoljni slovenski prostor. 